Check for updates

Cite this: Chem. Sci., 2019, 10, 4699

๑ All publication charges for this article have been paid for by the Royal Society of Chemistry

Received 23rd January 2019

Accepted 21st March 2019

DOI: $10.1039 / c 9 s c 00375 d$

rsc.li/chemical-science

\section{NIR-II light-modulated thermosensitive hydrogel for light-triggered cisplatin release and repeatable chemo-photothermal therapy $\dagger$}

\author{
Changping Ruan, (1) † Chanjuan Liu, Hailu Hu, Xiao-Lu Guo, Bang-Ping Jiang, \\ Hong Liang and Xing-Can Shen (DD *
}

Cisplatin is one of the most effective chemotherapeutic agents, although its clinical use is limited by severe nephrotoxicity. Multifunctional platform for spatiotemporally controlled delivery of cisplatin and multimodal synergistic therapy is highly desirable in antitumor research. Herein, for the first time, an injectable, NIR-II light-modulated and thermosensitive hydrogel is synthesized through supramolecular self-assembly of a conjugated polymer and $\alpha$-cyclodextrin. This hydrogel intrinsically features NIR responsive characteristics and thermo-responsive properties. The conjugated polymer (poly( $N$-phenylglycine)) not only tethers the poly(ethylene glycol) chains to enable the hydrogel formation, but also serves as the NIR-absorbing mediators. Accordingly, one particular benefit of this hydrogel is that its building blocks absorb NIR-II light and mediate the photothermal conversion itself, offering the important advantage of a prolonged retention time and thus permitting repeatable treatment upon a single-injection of this hydrogel. Under NIR-II laser irradiation, the localized photothermal effect not only ablates the highly metastatic triple-negative breast cancer (TNBC), but also triggers the on-demand cisplatin release through the thermo-responsive gel-sol transition, thus resulting in enhanced antitumor activity and reduced off-target toxicity. This work not only provides a novel multifunctional platform for NIRtriggered cisplatin release and chemo-photothermal combination therapy, but also presents a promising strategy for the rational design of NIR light-responsive hydrogels for the intervention of highly aggressive cancers.

\section{Introduction}

Cisplatin (cis-diamminedichloroplatinum(II), denoted as CDDP) is one of the most effective chemotherapeutic agents for the treatment of various cancers, although its clinical use is limited by severe renal toxicity and chemoresistance..$^{1-6}$ Effective accumulation of drugs in target sites at a sufficiently high concentration with less nonspecific distribution to normal organs is crucial for the clinical application of cisplatin, as well as for the other anticancer drugs..$^{4-8}$ Spatially and temporally controlled delivery of therapeutic agents could improve the cisplatin release to tumor sites, and therefore would enhance its antitumor efficiency and minimize its off-target toxicity. ${ }^{4-7}$ Moreover, owing to the aggressive and resistant nature of cancer, the complete eradication of cancer by using chemotherapy alone remains a challenge. The integration of two or more treatment

State Key Laboratory for Chemistry and Molecular Engineering of Medicinal Resources, School of Chemistry and Pharmaceutical Science, Guangxi Normal University, Guilin, 541004, P. R. China.E-mail: xcshen@mailbox.gxnu.edu.cn; hliang@gxnu.edu.cn

$\dagger$ Electronic supplementary information (ESI) available: Experimental section, characterization details, and additional figures. See DOI: 10.1039/c9sc00375d

$\ddagger$ These authors contributed equally to this work. modalities within one vehicle has demonstrated improved therapeutic efficacy relative to the use of each approach independently. ${ }^{8-15}$ Therefore, a combination of precise and controlled delivery of cisplatin with other treatment modality may further overcome the limitations of cisplatin and strengthen multiple advantages of these treatment modalities, and thus is highly desirable in clinical treatment of cancer. Among various combined therapy modalities, chemophotothermal synergistic therapy has proven to be a potent therapeutic approach for the treatment of various cancers. ${ }^{12-15}$ Furthermore, the extensively applied small-molecule therapeutic agents and nanosized drug delivery systems suffer from the disadvantages of rapid clearance from the body, short retention time, and insufficient dosage to the tumors, which requires multiple (intravenous or oral) administrations of therapeutic agents and therefore increases the patient's discomfort. ${ }^{7,16}$ Taken together, it's essential to construct multifunctional therapeutic systems that possess both the capability of spatiotemporally controlled delivery and multimodal synergistic therapy, as well as long-term retention in diseased sites.

Injectable hydrogels have been recognized as attractive platforms for local delivery of various therapeutic agents, as 
they provide high regional drug concentration, localized and sustained releasing characteristics, low systemic toxicities, minimal invasiveness, and long-term retention in diseased sites.,17-19 Compared with passive delivery via diffusion or degradation mechanisms, stimuli-responsive hydrogels in response to various internal or external triggers, such as light, $\mathrm{pH}$, enzymes, and temperature, deliver therapeutic agents in a more controllable manner., ${ }^{\mathbf{9} 2-24}$ Among the various stimuli, light affords near-instantaneous treatment with precise on and off spatiotemporal control in a noninvasive manner. Because of the good bio-safety and high transparency in the "biological window", the application of NIR light as a trigger to induce photothermal therapy (PTT) and on-demand delivery has gained particular interest., 22-32 More significantly, NIR-II window (1000-1400 nm) is more superior to the commonly utilized NIR-I window (750-1000 nm), due to the advantages of deeper depth of tissue penetration and higher maximum permissible exposure (MPE) to the laser. For NIR-II, the tissue penetration depth is expected to be maximal over the wavelength range $1000-1100 \mathrm{~nm}$, and the MPE is $1 \mathrm{~W} \mathrm{~cm}^{-2}$ at this spectral range. ${ }^{2-32}$ Consequently, the development of stimuliresponsive hydrogels responding to NIR light, especially NIRII light, is of great scientific significance and practical value. Traditional NIR light-responsive hydrogels usually had nanosized photothermal agents embedded in the matrices of thermosensitive hydrogels to mediate the photothermal conversion and trigger subsequent on-demand release through thermoinduced gel-sol transition. ${ }^{\mathbf{8 , 9}, 24}$ Some of these hydrogels are limited in use because the embedded nanosized NIR-absorbing materials may leach out, resulting in deteriorated photothermal effect and therefore inferior drug delivery ability. Previous works demonstrated that the backbone or building blocks of hydrogel could serve as photothermal mediators for PTT, and that the type of the hydrogel could prolong retention. ${ }^{\mathbf{1 6 , 3 3}}$ However, the lack of a reversible thermo-responsive phase transition of these hydrogels is a disadvantage that might compromise their application for NIR-triggered on-demand drug release.

We present herein the design and synthesis of an "all-in-one" multifunctional stimuli-responsive hydrogel that could overcome any of the aforementioned fundamental limitations. This new hydrogel intrinsically features NIR responsive characteristics and thermo-responsive properties in a single system. This superior hydrogel, composed of a conjugated polymer (poly $(\mathrm{N}$ phenylglycine), denoted hereafter as PNPG), poly(ethylene glycol) (PEG), and $\alpha$-cyclodextrin ( $\alpha$-CD) was fabricated through a supramolecular self-assembly strategy. Importantly, the PNPG not only tethered the PEG chains to enable the hydrogel formation, but also served as the NIR-absorbing mediators. Accordingly, one particular benefit of this hydrogel is that its building blocks (PNPG polymer) absorbed NIR-II light and mediated the photothermal conversion itself, offering the important advantage of a prolonged retention time and thus permitting repeatable treatment. To the best of our knowledge, this was the first example demonstrating that the building blocks of a hydrogel rather than of any exogenous nanostructures could respond to NIR-II laser. More importantly, the combination of NIR light-responsive characteristics and the thermo-responsive properties imparted to this hydrogel with the capability of NIR-triggered on-demand therapeutic drug release. This on-demand release ability efficiently enhanced the localized antitumor efficiency of cisplatin and minimized its off-target toxicity, both highly desirable characteristics in the clinical application of cisplatin. ${ }^{\mathbf{1 - 6}}$ Therefore, under low-powerdensity NIR-II laser irradiation, a localized photothermal effect induced by the hydrogel not only ablated the tumor cells, but also stimulated chemotherapy by enabling thermosensitive ondemand release of the chemotherapeutic drug of CDDP, which led to enhanced therapeutic efficacy compared with monotherapy (Fig. 1). Both in vitro and in vivo experiments demonstrated a remarkable synergistic therapeutic efficacy against the aggressive triple-negative breast cancer (TNBC). The present work not only provides a novel multifunctional platform for NIR-triggered cisplatin release and repeatable chemophotothermal combination therapy, but also presents a promising strategy for the rational design of NIR light-responsive hydrogels.

\section{Results and discussion}

\section{Synthesis and characterization}

With the aim of constructing the NIR light-responsive and thermosensitive supramolecular hydrogel, we first prepared the composite of PNPG and PEG (denoted as PNPG-PEG) via the polymerization of $N$-phenylglycine (NPG) in the presence of PEG
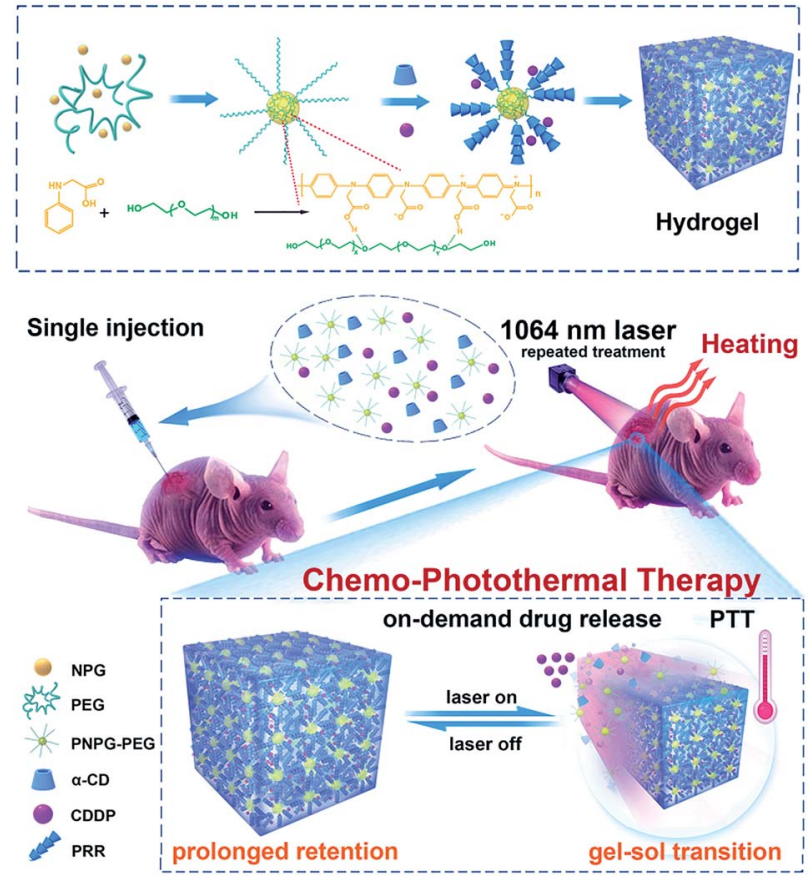

Fig. 1 Schematic illustration of the fabrication of the NIR-II lightresponsive hydrogel based on supramolecular self-assembly and its application for NIR-triggered cisplatin release and repeated chemophotothermal therapy based on "one injection, multiple treatment" strategy. 
(Fig. 1 and S1-S5, ESI $\dagger$ ). Dynamic light scattering (DLS) analysis and transmission electron microscopy (TEM) image revealed that the PNPG-PEG composite had a sea urchin-like structure with the size range of 400 to $900 \mathrm{~nm}$ (Fig. S4a, ESI $\dagger$ ). It is likely that the PEG chains were tethered by PNPG polymers through hydrogen-bond interactions and physical entanglement to form this sea urchin-like structure. Subsequently, the supramolecular hydrogel was prepared by mixing equal volumes of PNPGPEG (30 $\left.\mathrm{mg} \mathrm{mL}^{-1}\right)$ and $\alpha$-CD $\left(140 \mathrm{mg} \mathrm{mL}^{-1}\right)$ aqueous solution. $\alpha-\mathrm{CD}$ is a class of cyclic oligosaccharide that has molecularcompatible cavity. The exterior of its cavity is highly polar, because of the hydroxy groups, while the interior of its cavity is nonpolar. Upon the mixing of PNPG-PEG and $\alpha$-CD solution, the free ends of PEG chains threaded into a series of $\alpha$-CD rings via host-guest inclusion to form pseudopolyrotaxanes (PPRs, as illustrated in Fig. 1). Then, the hydrogen-bond interactions between the adjacent threaded $\alpha$-CDs led to the microcrystalline aggregation and thus provided the physical cross-links that promoted the formation of the supramolecular hydrogel (denoted as PNPG-PEG/ $\alpha$-CD hydrogel, Fig. 1 and 2a)..$^{\mathbf{2 4 3 4 - 3 6}}$

$\mathrm{X}$-ray diffraction (XRD) analysis proved the formation of PPRs, the peak located at $19.8^{\circ}$ in the XRD pattern of PNPGPEG/ $\alpha$-CD hydrogel was attributed to the column crystalline complex of PEG/ $\alpha-\mathrm{CD}$ inclusions (Fig. S6, ESI $\dagger$ ). ${ }^{34-36}$ Scanning electron microscopy (SEM) confirmed the porous structure of the dried gel network (Fig. S7, ESI $\dagger$ ). Also, formation of the hydrogel was confirmed by a rheology test, as evidenced by development and crosslink of storage $\left(G^{\prime}\right)$ and loss $\left(G^{\prime \prime}\right)$ moduli within several minutes (Fig. S8, ESI $\dagger$ ). ${ }^{19,24}$ As a control, the direct mixing of PNPG, PEG, and $\alpha$-CD did not generate a hydrogel, highlighting the necessity of tethering the PEG chains to form the hydrogel (Fig. S9, ESI $\dagger$ ). Specifically, the tethering of the PEG chains by PNPG was able to prevent the complete inclusion of $\alpha$ CD on the PEG chains and additionally provide stable and constant physical cross-links, both of which were beneficial for the hydrogel formation. ${ }^{36}$ Due to the de-threading and rethreading of $\alpha$-CD from PEG chains upon the change of temperature, the hydrogel underwent reversible gel-sol transitions at $52{ }^{\circ} \mathrm{C}$, implying its thermo-sensitive property (Fig. S10, ESI $\dagger) .{ }^{24}$ The character of thermo-sensitive and reversible gel-sol transition made this hydrogel suitable for locally controlled delivery of drugs.

\section{Photothermal performance}

The ultraviolet-visible-near-infrared (UV-vis-NIR) absorption spectrum was performed to probe the optical properties and the potential for photothermal conversion (Fig. 2b, S11 and S12, ESI $\dagger$ ). The PNPG-PEG composite displayed strong and broad a

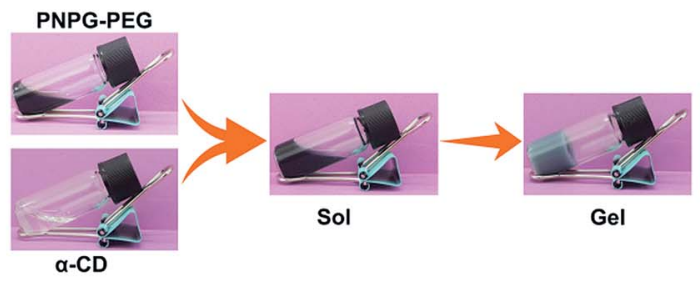

C

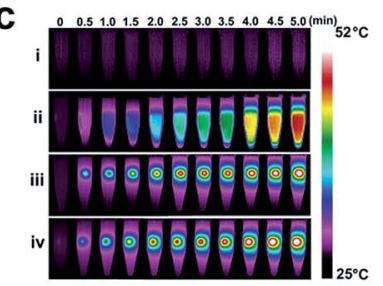

e

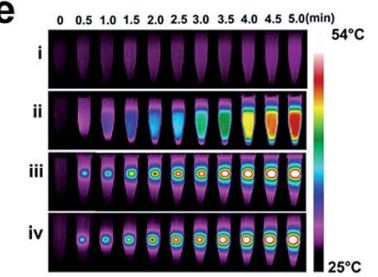

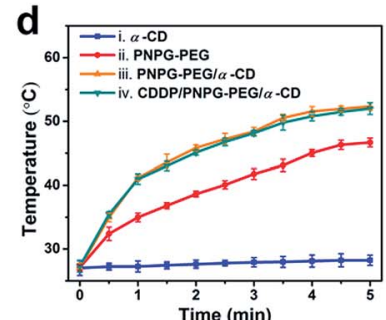

f

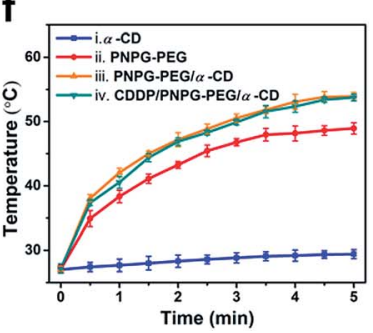

b

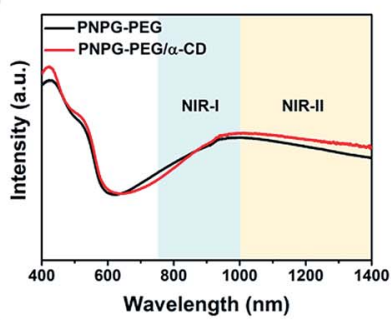

g

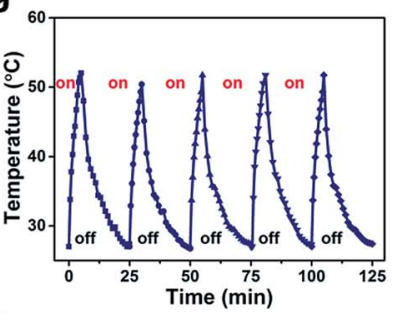

h

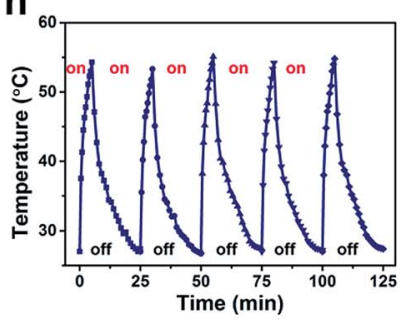

Fig. 2 Synthesis and characterization. (a) The formation of PNPG-PEG/ $\alpha-C D$ hydrogel upon the mixing of PNPG-PEG (30 mg $\mathrm{mL}^{-1}$ ) and $\alpha-C D$ aqueous solution $\left(140 \mathrm{mg} \mathrm{mL}^{-1}\right.$ ) at room temperature; (b) normalized UV-vis-NIR absorbance spectra of PNPG-PEG/ $\alpha$-CD hydrogel, compared with PNPG-PEG (in water, $15 \mathrm{mg} \mathrm{mL}^{-1}$ ); (c and e) thermo-graphic images of $\alpha$-CD (in water, $70 \mathrm{mg} \mathrm{mL}^{-1}$ ), PNPG-PEG (in water, $15 \mathrm{mg} \mathrm{mL}^{-1}$ ), PNPG-PEG/ $\alpha-C D$ hydrogel and CDDP/PNPG-PEG/ $\alpha-C D$ hydrogel upon exposure to NIR laser $\left(5\right.$ minutes, $\left.0.5 \mathrm{~W} \mathrm{~cm}^{-2}\right)$ and $(\mathrm{d}$ and $\mathrm{f})$ the corresponding temperature evolutions with ( $c$ and d) $808 \mathrm{~nm}$ and (e and f) $1064 \mathrm{~nm}$ irradiation; ( $g$ and h) temperature profiles of PNPG-PEG/ $\alpha$ $\mathrm{CD}$ hydrogel for five successive cycles of laser on (5 minutes, $0.5 \mathrm{~W} \mathrm{~cm}^{-2}$ ) and off processes under (g) $808 \mathrm{~nm}$ and (h) $1064 \mathrm{~nm}$ laser irradiation. 
absorption that covered both NIR-I and NIR-II regions (from $750 \mathrm{~nm}$ to $1400 \mathrm{~nm}$ ). Control experiments proved that it was the doping effect that shifted the absorbance (associated with the charge transfer between quinoid and benzenoid rings) of PNPG from the visible light region to the NIR spectral range, leading to this strong and broad absorption ranging from the NIR-I to NIRII regions (see Fig. S11 and S12 for the detailed analysis, ESI $\dagger)^{37-39}$ The PNPG-PEG/ $\alpha$-CD hydrogel had strong and broad absorption that covered both NIR-I and NIR-II regions (from $750 \mathrm{~nm}$ to $1400 \mathrm{~nm}$ ), similar as that of PNPG-PEG composite (Fig. 2b).

Note that the PNPG-PEG composite acted not only as the building blocks for the hydrogel formation, but also as photothermal conducting mediators. The high photothermal conversion efficiency of PNPG-PEG $(40 \%$ and $41 \%$ under $808 \mathrm{~nm}$ and $1064 \mathrm{~nm}$ laser irradiation, respectively, Fig. S13$\mathrm{S} 15, \mathrm{ESI} \dagger$ ) enabled the potential of the PNPG-PEG/ $\alpha$-CD hydrogel for PTT. As shown in Fig. 2c-f, being exposed to laser irradiation for 5 minutes at $0.5 \mathrm{~W} \mathrm{~cm}{ }^{-2}$, the temperature increments of the PNPG-PEG/ $\alpha$-CD hydrogel were $25.2{ }^{\circ} \mathrm{C}$ (808 nm laser) and $26.4{ }^{\circ} \mathrm{C}$ (1064 nm laser), indicating the good photothermal effect of this hydrogel. It is worth noting that the resultant temperature of the hydrogel $\left(52.3{ }^{\circ} \mathrm{C}\right.$ for the $808 \mathrm{~nm}$ laser, $53.9^{\circ} \mathrm{C}$ for the $1064 \mathrm{~nm}$ laser) was slightly higher than its gel-sol transition temperature, warranting its potential application in NIR-triggered therapeutic drug release. The obvious temperature increments of the PNPG-PEG composite and the slight temperature increase of the $\alpha$-CD solution under the same test conditions confirmed that the heat generation ability of PNPG-PEG/ $\alpha$-CD hydrogel indeed originated from the PNPGPEG composite. This feature offered significant advantages over traditional NIR responsive hydrogels, which used the loaded exogenous NIR-absorbing nanostructures to mediate photothermal conversion., ${ }^{\mathbf{8 , 9}, 24}$ Furthermore, the photothermal performance of the PNPG-PEG/ $\alpha$-CD hydrogel remained constant after five successive cycles of laser on and off processes (Fig. $2 \mathrm{~g}$ and $\mathrm{h}$ ), permitting the potential of the PNPG-PEG/ $\alpha$-CD hydrogel to be a durable photothermal agent for repeated treatment. ${ }^{29-32}$

\section{Photothermal conversion capability under tissue-penetrating NIR laser}

With its lower absorption and light scattering by tissues, the NIR-II spectral window is known to offer higher tissuepenetration depth than the frequently utilized NIR-I window, thus usually giving rise to a better photothermal effect. ${ }^{29-32}$ To prove this concept, we evaluated the deep tissue photothermal conversion capability in both the NIR-I and NIR-II windows by detecting the temperature elevations after tissue penetration under laser irradiations (Fig. 3a). Chicken breast tissues of varying thicknesses were applied as model biological tissues (Fig. S16, ESI†). Owing to the absorption and scattering of light by the tissues, the temperature elevations of hydrogel exposed to tissue-penetrating NIR laser gradually decreased as the thickness of the tissue increased (Fig. 3b). Still, the temperature elevations under the $1064 \mathrm{~nm}$ laser were higher than these
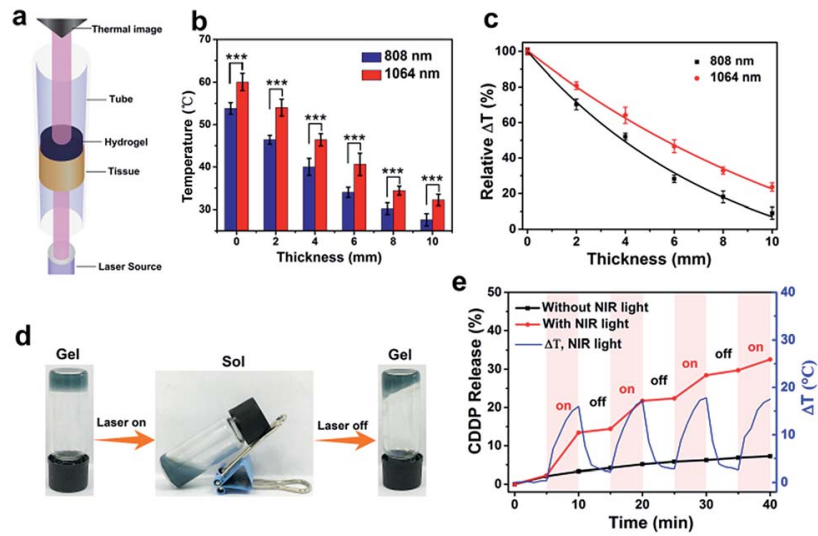

Fig. 3 Tissue-penetration capability and on-demand cisplatin release. (a) Schematic diagram for detecting tissue-penetration capability of NIR laser; (b) temperatures of PNPG-PEG/ $\alpha-C D$ hydrogel exposed to tissue-penetrating NIR-I $(808 \mathrm{~nm})$ and NIR-II (1064 nm) lasers (5 minutes, $0.5 \mathrm{~W} \mathrm{~cm}^{-2}$; ***P<0.001); (c) normalized temperature elevations of PNPG-PEG/ $\alpha-C D$ hydrogel exposed to tissue-penetrating lasers; (d) the thermo-sensitive and reversible gel-sol transition of the hydrogel under $1064 \mathrm{~nm}$ laser irradiation; (e) release of CDDP from the hydrogel under periodic irradiation and the corresponding photo-triggered temperature increase $\left(1064 \mathrm{~nm}\right.$ laser, $0.5 \mathrm{~W} \mathrm{~cm}^{-2}$, concentration of CDDP: $0.5 \mathrm{mg} \mathrm{mL}^{-1}$ ).

under the $808 \mathrm{~nm}$ laser, indicating the better photothermal performance in the NIR-II window. We also normalized the temperature increases measured at different tissue depths with respect to the temperature increase that occurred in the absence of any tissue, and the resultant data were plotted as a function of tissue thickness (Fig. 3c). With the increase of tissue depth, the attenuation rate of temperature elevation with the $1064 \mathrm{~nm}$ laser was slower than that of with the $808 \mathrm{~nm}$ laser, indicating the better tissue penetration ability in the NIR-II windows. Considering the advantages of better tissue penetration ability and higher MPE to the laser, for subsequent in vitro and in vivo investigations, we chose to use the $1064 \mathrm{~nm}$ laser as the irradiation light.

\section{NIR-triggered cisplatin release in vitro}

The as-prepared hydrogel underwent thermo-induced reversible gel-sol transition under NIR laser irradiation (Fig. 3d and S17, ESI $\dagger$ ), and thus should be capable of NIR-triggered therapeutic drug release. ${ }^{924}$ To verify this hypothesis, the in vitro release profile of CDDP from the hydrogel under $1064 \mathrm{~nm}$ laser irradiation was investigated (Fig. 3e and S18, ESI $\dagger$ ). The clinical benefit of CDDP is known to be compromised by its dosedependent toxicity and chemoresistance. ${ }^{1-6}$ We assumed that encapsulating CDDP into this hydrogel and then triggering its localized on-demand release by using NIR laser could improve the CDDP delivery to tumor sites, and therefore would enhance its antitumor efficiency and minimize its off-target toxicity. The CDDP-laden hydrogel (denoted as CDDP/PNPG-PEG/ $\alpha$-CD hydrogel) was prepared by dissolving the CDDP into the PNPGPEG aqueous solution, followed by mixing the obtained solution with $\alpha$-CD solution. As expected, CDDP was released from 
the CDDP/PNPG-PEG/ $\alpha$-CD hydrogel at the rate of over $11 \%$ upon the first cycle of laser irradiation for 5 minutes. When the laser irradiation was ceased, the release rate of CDDP was considerably slower. During the second cycle of laser exposure, the release of CDDP recommenced and increased from $14 \%$ to $22 \%$ within 5 minutes. After 4 cycles of laser irradiation, the total release of CDDP was 33\%, whereas the control group without laser irradiation only released $7 \%$ of the loaded CDDP. This on-demand release characteristic will facilitate CDDP release at target sites at a sufficiently high concentration with less nonspecific distribution to normal organs.

\section{In vitro therapeutic efficacy assessments}

The good photothermal effect and the NIR light-triggered drug release of the hydrogel supported our efforts to evaluate its applicability for combined PTT and chemotherapy. Before further cell experiments, standard methyl thiazolyl tetrazolium (MTT) assay and the Live/Dead staining method were performed to assess the biocompatibility of this hydrogel (Fig. 4a and S19, ESI $\dagger$ ). As shown in Fig. 4a, the high cell viability of L929 mouse fibroblast cells (noncancer cell), MCF-7 cells (human breast cancer), and MDA-MB-231 cells (triple negative breast cancer) even at the concentration up to $10 \mathrm{~g} \mathrm{~L}^{-1}$ indicated that the PNPG-PEG/ $\alpha$-CD hydrogel alone had negligible cellkilling ability towards these cells. The good biocompatibility of this hydrogel made it suitable for the following biological
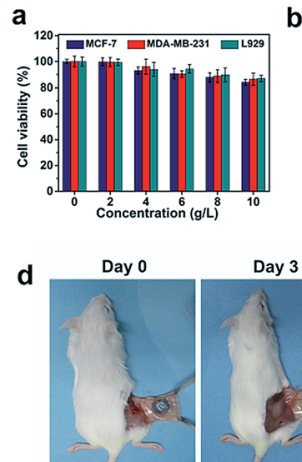

e

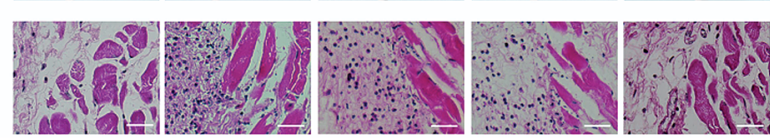

Fig. 4 The evaluation of in vitro antitumor efficacy and in vivo gelation and degradability. (a) Viability of L929 cells, MCF-7 cells, and MDA-MB231 cells treated with varying concentrations of PNPG-PEG/ $\alpha-C D$ hydrogel (48 hours, without laser irradiation); (b) confocal images of calcein $A M(1.6 \%$, for live cells, green fluorescence) and propidium iodide (PI, $2 \%$, for dead cells, red fluorescence) co-stained MDA-MB231 cells after incubation with PNPG-PEG/ $\alpha$-CD hydrogel $\left(8 \mathrm{mg} \mathrm{mL}^{-1}\right)$ and exposure to $1064 \mathrm{~nm}$ laser for different durations $(0,1,3,5$ minutes; at $0.5 \mathrm{~W} \mathrm{~cm}^{-2}$, scale bar: $400 \mu \mathrm{m}$ ); (c) viability of MDA-MB231 cells treated with various administrations; (d) photographs of the subcutaneously injected hydrogels at different time intervals postinjection, and (e) H\&E staining of the corresponding subcutaneous tissues surrounding the hydrogel (the nuclei were stained bluish violet, and the cytoplasm and extracellular matrix were stained pink. Scale bar: $40 \mu \mathrm{m})$. applications. The aggressive cisplatin-resistant MDA-MB-231 breast cancer cells were chosen as a model for in vitro and in vivo assessments (Fig. S20-S22, ESI $\dagger$ ). First, the photothermal cytotoxicity of PNPG-PEG/ $\alpha$-CD hydrogel was evaluated by using the Live/Dead staining method. Before being exposed to laser, almost all the cells incubated with PNPG-PEG/ $\alpha$-CD hydrogel were colored green without the presence of dead cells (red). When exposed to $1064 \mathrm{~nm}$ laser irradiation at $0.5 \mathrm{~W} \mathrm{~cm}^{-2}$, cells in the central region within the laser irradiation spot were stained in red, indicating the death of cells (Fig. 4b). As irradiation was prolonged, the area of the dead cell cycle (red) was enlarged dramatically, indicating the time-dependent cellkilling ability. A remarkable decline of cell viability was observed when the irradiation was extended to 5 minutes, highlighting the good photothermal effect induced by PNPGPEG/ $\alpha$-CD hydrogel under $1064 \mathrm{~nm}$ laser.

Encouraged by the exciting outcome of photothermal treatment, we next evaluated the in vitro antitumor activity of combined PTT and chemotherapy (Fig. 4c). Treatment of MDAMB-231 cells with CDDP/PNPG-PEG/ $\alpha$-CD hydrogel followed by $1064 \mathrm{~nm}$ laser irradiation reduced cell viability to less than $10 \%$, whereas the cell viability of MDA-MB-231 cells treated with PNPG-PEG/ $\alpha$-CD hydrogel alone under the same test condition was approximately $90 \%$ (Fig. 4a). The viability of control groups was 30\% (PNPG-PEG/ $\alpha$-CD hydrogel under $1064 \mathrm{~nm}$ laser), 46\% (free CDDP), and $60 \%$ (CDDP/PNPG-PEG/ $\alpha$-CD hydrogel without laser). These observations indicated that remarkably enhanced cell killing effect of CDDP/PNPG-PEG/ $\alpha$-CD hydrogel followed by $1064 \mathrm{~nm}$ laser irradiation was attributed to both the cytotoxic effect of released CDDP and the photothermal effect mediated by the hydrogel.

\section{In vivo gelation and in vivo degradability}

Next, in vivo formation and degradation of the hydrogel were examined to assess its feasibility as an injectable system for in vivo treatment (Fig. 4d). Briefly, $200 \mu \mathrm{L}$ precursor solutions of PNPG-PEG/ $\alpha$-CD (the mixture of $100 \mu \mathrm{L}$ PNPG-PEG $(30 \mathrm{mg}$ $\left.\mathrm{mL}^{-1}\right)$ and $100 \mu \mathrm{L} \alpha$-CD $\left(140 \mathrm{mg} \mathrm{mL}^{-1}\right)$ ) were subcutaneously injected into the Kunming mice. The mice were sacrificed at 30 minutes, 3 days, 7 days, 14 days, and 28 days post-injection to observe the gel morphology. As expected, the hydrogel was successfully formed in subcutaneous tissues of mice. The integrity of the hydrogel remained up to 7 days, whereas its overall size was gradually decreased. The persistent retention of this hydrogel within the injection site is beneficial for repeated PTT and the avoidance of multiple injections, thus offering significant advantages over nanostructured photothermal agents. ${ }^{7,9,16}$ At two weeks post-injection, the hydrogel was almost completely degraded with few residual deposits, indicating its degradability in vivo. The in vivo degradation of the hydrogel was probably related to the de-threading of $\alpha$-CD from PEG chains and the subsequent degradation of the PNPG-PEG composite by the oxidative environment in vivo (Fig. S23, ESI $\dagger$ ). ${ }^{40}$ Note that the degradation of the hydrogel ensures that the implanted composite will be eliminated harmlessly from the body within a reasonable period of time after finishing the 
therapy. ${ }^{19}$ Haematoxylin and eosin (H\&E) staining of the subcutaneous tissues surrounding the hydrogel was performed to estimate the biocompatibility of the hydrogel in vivo (Fig. 4e). At 3 days post-injection, infiltration of inflammatory cells was observed because of the inflammatory response. As the hydrogels degraded gradually, the number of inflammatory cells was reduced and the inflammation was gradually eliminated. After the complete degradation of the hydrogel (28 days postinjection), the inflammation subsidized without causing obvious tissue damage, implying the good biocompatibility of the hydrogel in vivo.

\section{In vivo therapeutic efficacy of chemo-photothermal therapy}

Having demonstrated the marked in vitro antitumor activity, the injectability, and the in vivo gelation and degradability of the hydrogel, we next probed the in vivo therapeutic efficacy of chemo-photothermal therapy induced by this multifunctional hydrogel. MDA-MB-231 tumor-bearing mice were randomly divided into eight groups and treated with various administrations (intratumoral injection of saline, free CDDP, PNPG-PEG/ $\alpha$-CD hydrogel, and CDDP/PNPG-PEG/ $\alpha$-CD hydrogel; followed with or without laser irradiation) (Fig. 5 and S24, ESI $\dagger$ ). For the groups exposed to laser, a total of four cycles of laser irradiation (at 0, 2, 4 and 6 days following injections, 5 minutes each cycle) was carried out. When exposed to laser irradiation, the groups injected with a single dose of photothermal hydrogel
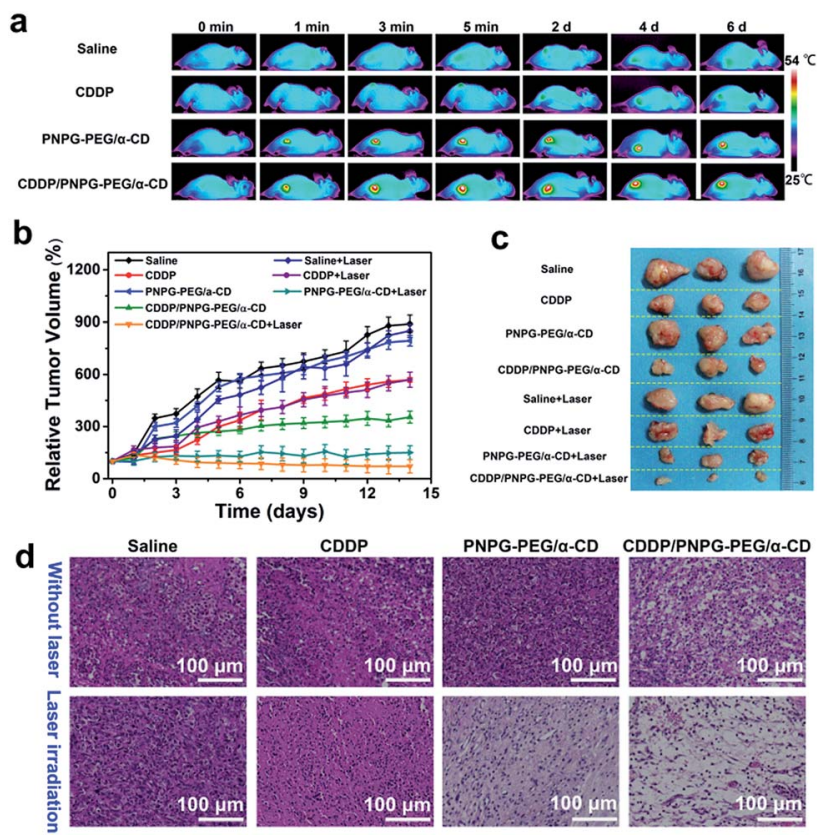

Fig. 5 The evaluation of in vivo antitumor efficacy. (a) Infrared thermal images of mice under $1064 \mathrm{~nm}$ laser irradiation (irradiation time: 5 minutes; laser power: $0.5 \mathrm{~W} \mathrm{~cm}^{-2}$ ); (b) time-dependent tumor growth curves; (c) digital photos of extracted tumors; (d) H\&E staining of the tumor tissues, the tumors of the group treated with CDDP/PNPG$\mathrm{PEG} / \alpha-C D$ hydrogel plus laser was severely damaged (the nuclei were stained bluish violet, and the cytoplasm and extracellular matrix were stained pink). ${ }^{42}$
(PNPG-PEG/ $\alpha$-CD or CDDP/PNPG-PEG/ $\alpha$-CD) showed rapid temperature rise in the tumors and resulted in a tumor temperature of $54{ }^{\circ} \mathrm{C}$ in 5 minutes, indicating the good photothermal effect induced by the hydrogel (Fig. 5a and S25, ESI $\dagger$ ). Thus, loading of CDDP into the hydrogel had no adverse effect on the temperature elevation. Due to the lack of photothermal agents, negligible temperature increase was observed for the groups injected with saline and free CDDP. Notably, the hydrogel maintained excellent photothermal activity even at 6 days post-injection, thus permitting repeated PTT. As shown in Fig. 5a, during the fourth cycle of laser exposure, the resulting tumor temperature was still higher than $50{ }^{\circ} \mathrm{C}$, confirming the feasibility of using this hydrogel for repeated treatment upon a single injection.

The tumor sizes and body weight were measured every day during the treatments to evaluate therapeutic efficiency and side effects. It is reported that cisplatin resistance often leads to therapeutic failure. ${ }^{1-3,41}$ In the present work, free CDDP (with and without laser) inhibited the tumor growth at the first 3 days; but thereafter, rapid growth of tumors was observed (Fig. 5b). A combination of NIR-triggered delivery of cisplatin with PTT may overcome the limitation of cisplatin and strengthen the dual advantages of chemotherapy and PTT. As shown in Fig. 5b, treatment with CDDP/PNPG-PEG/ $\alpha$-CD followed by $1064 \mathrm{~nm}$ laser irradiation suppressed the tumor growth more efficiently than in the other groups, which should be a result of the combination chemotherapy and PTT. In comparison with free CDDP treated groups, the tumor growth rate of the group treated with CDDP/PNPG-PEG/ $\alpha$-CD hydrogel was much slower, indicative of a localized and sustained release of CDDP from the hydrogel which could efficiently enhance the antitumor activity of CDDP. For the group treated with PNPG-PEG/ $\alpha$-CD hydrogel followed by laser irradiation, the tumors showed negligible growth, confirming the good antitumor efficiency of the PTT. In striking contrast, treatments with saline, saline plus laser, and PNPG-PEG/ $\alpha$-CD hydrogel alone had negligible inhibitory effect on tumor growth. This observation made clear that neither PNPG-PEG/ $\alpha$-CD hydrogel itself nor the NIR irradiation alone could inhibit the tumor growth. The tumors excised at the end of treatments also confirmed that the CDDP/PNPG-PEG/ $\alpha-C D$ hydrogel plus laser group resulted in the smallest tumors when compared to all the controls, indicating the most significant antitumor efficacy of combination therapy (Fig. 5c and S26, $\mathrm{ESI}, \dagger * * * P<0.001, n=3)$. Moreover, histopathological analysis of the tumor tissues was performed to evaluate the anti-tumor activity at cellular level (Fig. 5d). Clearly, the combination therapy meditated by CDDP/PNPG-PEG/ $\alpha$-CD hydrogel and laser resulted in the most extensive apoptotic and necrotic cells in comparison with other control groups, showing obvious nuclear damage, cell shrinkage and separation. However, no noticeable apoptosis could be observed for the three groups treated with saline, saline plus laser, and PNPG-PEG/ $\alpha$-CD hydrogel (without laser irradiation). For the groups treated with CDDP/PNPG-PEG/ $\alpha$-CD hydrogel (without laser, chemotherapy alone) or PNPG-PEG/ $\alpha$-CD hydrogel plus laser (photothermal therapy alone), the apoptosis was less severe than that of the CDDP/PNPG-PEG/ $\alpha$-CD hydrogel plus laser group, but more 
severe than the groups treated with free CDDP. Additionally, of particular importance is the low power density of laser irradiation in this work $\left(0.5 \mathrm{~W} \mathrm{~cm}^{-2}\right)$, which is far below the MPE for skin exposure in the NIR-II window $\left(1 \mathrm{~W} \mathrm{~cm}^{-2}\right)$ and therefore warrants potential for clinical translation. ${ }^{29-32}$

No apparent body weight loss was observed during the therapeutic period for the groups treated with CDDP/PNPGPEG/ $\alpha$-CD hydrogel (with and without laser), indicating reduced side effects related to the toxicity of CDDP (Fig. S27, ESI $\dagger$ ). In contrast, treatment with free CDDP led to obvious loss of body weight, implying the side effects caused by the toxicity of CDDP..$^{1-6}$ The other four control groups (treated with saline, saline plus laser, PNPG-PEG/ $\alpha$-CD hydrogel alone, and PNPGPEG/ $\alpha$-CD hydrogel plus laser) also showed steady body weight increases. Moreover, histopathological analysis revealed that the combination therapy meditated by CDDP/PNPG-PEG/ $\alpha$-CD hydrogel and laser did not induce obvious side effect to the major organs. As shown in Fig. S28 (ESI), † no detectable lesions, such as necrosis, hydropic degeneration, inflammatory, pulmonary fibrosis, were observed in the sections of the major organs tissues (including heart, liver, spleen, lung and kidney), confirming that the PNPG-PEG/ $\alpha$-CD hydrogel was biocompatible and the toxicity of CDDP was reduced by encapsulating CDDP into this hydrogel (Fig. S29, ESI $\dagger$ ). Owing to the nephrotoxicity of CDDP, the kidneys from the groups treated with free CDDP showed obvious disappearance of renal capsule cavity (marked by arrows, see Fig. S28, ESI $\dagger$ ). ${ }^{1-6}$ The clinical benefit of CDDP is known to be compromised by its severe nephrotoxicity and chemoresistance. ${ }^{1-6}$ In the present work, a combination of precise and controlled delivery of cisplatin with simultaneous PTT effectively overcame the limitations of cisplatin and strengthened dual advantages of both chemotherapy and PTT, thus resulting in enhanced antitumor efficiency and reduced side effects.

\section{Conclusions}

In conclusion, we have successfully designed an "all-in-one" multifunctional stimuli-responsive hydrogel for NIR-triggered delivery of cisplatin and repeatable chemo-photothermal combination therapy. The NIR laser-modulated photothermal effect of this novel hydrogel not only mediates photothermal ablation of cancer cells, but also triggers thermo-induced transition of gel-sol, affording subsequent on-demand cisplatin release. As a consequence, combining PTT with simultaneous NIR laser-activated chemotherapy in the present work provided an opportunity for efficient eradication of aggressive tumors, leading to significantly enhanced antitumor efficiency. Importantly, this work provides a potential new multifunctional platform for NIR-triggered cisplatin release and repeatable chemo-photothermal therapy for the intervention of TNBC or other highly aggressive cancers. Furthermore, this design strategy presents a promising strategy for the rational design of NIR light-responsive hydrogels and thus may help expand the stimuli-responsive hydrogel reservoirs.

\section{Ethical statement}

All animal experimental procedures were carried out in compliance with the policies of the Animal Care and Use Committee of Guangxi Medical University (Nanning, China) and were approved by the local Animal Care and Use Committee.

\section{Conflicts of interest}

There are no conflicts to declare.

\section{Acknowledgements}

Financial support by National Natural Science Foundation of China (21671046, 21502028, 51762007 and 51562001), Natural Science Foundation of Guangxi Province (2017GXNSFGA198004, 2017GXNSFBA198208, 2018GXNSFFA281004 and AD 17129007), and the project of State Key Laboratory for Chemistry and Molecular Engineering of Medicinal Resources (Guangxi Normal University) (CMEMR2018-C5 and CMEMR2018-C25) is gratefully acknowledged.

\section{Notes and references}

1 L. Kelland, Nat. Rev. Cancer, 2007, 7, 573-584.

2 N. Wang, Z. Wang, Z. Xu, X. Chen and G. Zhu, Angew. Chem., Int. Ed., 2018, 57, 3426-3430.

3 J. Li, Y. Kuang, J. Shi, J. Zhou, J. E. Medina, R. Zhou, D. Yuan, C. Yang, H. Wang, Z. Yang, J. Liu, D. M. Dinulescu and B. Xu, Angew. Chem., Int. Ed., 2015, 54, 13307-13311.

4 S. Yu, D. Zhang, C. He, W. Sun, R. Cao, S. Cui, M. Deng, Z. Gu and X. Chen, Biomacromolecules, 2017, 18, 4341-4348.

5 H. Ma, C. He, Y. Cheng, Z. Yang, J. Zang, J. Liu and X. Chen, ACS Appl. Mater. Interfaces, 2015, 7, 27040-27048.

6 S. Dhar, N. Kolishetti, S. J. Lippard and O. C. Farokhzad, Proc. Natl. Acad. Sci. U. S. A., 2011, 108, 1850-1855.

7 M. Karimi, P. Sahandi Zangabad, S. Baghaee-Ravari, M. Ghazadeh, H. Mirshekari and M. R. Hamblin, J. Am. Chem. Soc., 2017, 139, 4584-4610.

8 M. Qiu, D. Wang, W. Liang, L. Liu, Y. Zhang, X. Chen, D. K. Sang, C. Xing, Z. Li, B. Dong, F. Xing, D. Fan, S. Bao, H. Zhang and Y. Cao, Proc. Natl. Acad. Sci. U. S. A., 2018, 115, 501-506.

9 Y. Wu, H. Wang, F. Gao, Z. Xu, F. Dai and W. Liu, Adv. Funct. Mater., 2018, 28, 1801000.

10 Z. Fangyuan, F. Bing, W. Tingting, W. Dangge, M. Qingshuo, Z. Jianfeng, Z. Zhiwen, W. Siling, Y. Haijun and L. Yaping, Adv. Funct. Mater., 2017, 27, 1606530.

11 J. Bai, Y. Liu and X. Jiang, Biomaterials, 2014, 35, 5805-5813. 12 H. Liu, D. Chen, L. Li, T. Liu, L. Tan, X. Wu and F. Tang, Angew. Chem., Int. Ed., 2011, 50, 891-895.

13 L. Zhang, Y. Chen, Z. Li, L. Li, P. Saint-Cricq, C. Li, J. Lin, C. Wang, Z. Su and J. I. Zink, Angew. Chem., Int. Ed., 2016, 55, 2118-2121.

14 J.-O. You, P. Guo and D. T. Auguste, Angew. Chem., Int. Ed., 2013, 52, 4141-4146. 
15 Y. Wang, K. Wang, J. Zhao, X. Liu, J. Bu, X. Yan and R. Huang, J. Am. Chem. Soc., 2013, 135, 4799-4804.

16 C. W. Hsiao, E. Y. Chuang, H. L. Chen, D. Wan, C. Korupalli, Z. X. Liao, Y. L. Chiu, W. T. Chia, K. J. Lin and H. W. Sung, Biomaterials, 2015, 56, 26-35.

17 Y. Li, J. Rodrigues and H. Tomas, Chem. Soc. Rev., 2012, 41, 2193-2221.

18 L. Yu and J. Ding, Chem. Soc. Rev., 2008, 37, 1473-1481.

19 C. Wang, X. Wang, K. Dong, J. Luo, Q. Zhang and Y. Cheng, Biomaterials, 2016, 104, 129-137.

20 N. Oliva, J. Conde, K. Wang and N. Artzi, Acc. Chem. Res., 2017, 50, 669-679.

21 B. P. Purcell, D. Lobb, M. B. Charati, S. M. Dorsey, R. J. Wade, K. N. Zellars, H. Doviak, S. Pettaway, C. B. Logdon, J. A. Shuman, P. D. Freels, J. H. Gorman III, R. C. Gorman, F. G. Spinale and J. A. Burdick, Nat. Mater., 2014, 13, 653661.

22 X. Xu, Z. Huang, X. Zhang, S. He, X. Sun, Y. Shen, M. Yan and C. Zhao, ACS Appl. Mater. Interfaces, 2017, 9, 20361-20375.

23 J. Hu, Y. Chen, Y. Li, Z. Zhou and Y. Cheng, Biomaterials, 2017, 112, 133-140.

24 X. Wang, C. Wang, Q. Zhang and Y. Cheng, Chem. Commun., 2016, 52, 978-981.

25 E. R. Ruskowitz and C. A. DeForest, Nat. Rev. Mater., 2018, 3, 17087.

26 S. Mura, J. Nicolas and P. Couvreur, Nat. Mater., 2013, 12, 991-1003.

27 Y. Wang, M. S. Shim, N. S. Levinson, H.-W. Sung and Y. Xia, Adv. Funct. Mater., 2014, 24, 4206-4220.

28 Z. Zhang, J. Wang, X. Nie, T. Wen, Y. Ji, X. Wu, Y. Zhao and C. Chen, J. Am. Chem. Soc., 2014, 136, 7317-7326.
29 Y. Jiang, J. Li, X. Zhen, C. Xie and K. Pu, Adv. Mater., 2018, 30, 1705980.

30 X. Wang, Y. Ma, X. Sheng, Y. Wang and H. Xu, Nano Lett., 2018, 18, 2217-2225.

31 H. Lin, S. Gao, C. Dai, Y. Chen and J. Shi, J. Am. Chem. Soc., 2017, 139, 16235-16247.

32 M.-F. Tsai, S.-H. G. Chang, F.-Y. Cheng, V. Shanmugam, Y.-S. Cheng, C.-H. Su and C.-S. Yeh, ACS Nano, 2013, 7, 5330-5342.

33 X. Wang, C. Wang, X. Wang, Y. Wang, Q. Zhang and Y. Cheng, Chem. Mater., 2017, 29, 1370-1376.

34 G. Chen and M. Jiang, Chem. Soc. Rev., 2011, 40, 2254-2266.

35 Q. Lin, Y. Yang, Q. Hu, Z. Guo, T. Liu, J. Xu, J. Wu, T. B. Kirk, D. Ma and W. Xue, Acta Biomater., 2017, 49, 456-471.

36 J. Yu, W. Ha, J. N. Sun and Y. P. Shi, ACS Appl. Mater. Interfaces, 2014, 6, 19544-19551.

37 J. Yang, J. Choi, D. Bang, E. Kim, E.-K. Lim, H. Park, J.-S. Suh, K. Lee, K.-H. Yoo, E.-K. Kim, Y.-M. Huh and S. Haam, Angew. Chem., Int. Ed., 2011, 50, 441-444.

38 B.-P. Jiang, L. Zhang, X.-L. Guo, X.-C. Shen, Y. Wang, Y. Zhu and H. Liang, Small, 2017, 13, 1602496.

39 B.-P. Jiang, L. Zhang, Y. Zhu, X.-C. Shen, S.-C. Ji, X.-Y. Tan, L. Cheng and H. Liang, J. Mater. Chem. B, 2015, 3, 3767-3776.

40 Y. Liu, K. Ai, J. Liu, M. Deng, Y. He and L. Lu, Adv. Mater., 2013, 25, 1353-1359.

41 J.-H. Lee, J.-W. Chae, J. K. Kim, H. J. Kim, J. Y. Chung and Y.-H. Kim, J. Controlled Release, 2015, 215, 82-90.

$42 \mathrm{X} . \mathrm{Wu}, \mathrm{Y} . \mathrm{Wu}, \mathrm{H}$. Ye, S. Yu, C. He and X. Chen, J. Controlled Release, 2017, 255, 81-93. 